\title{
PNEUMOTHORAX - RISK FACTORS IN NEWBORN INFANTS
}

\author{
Aniko Manea ${ }^{1,2}$, Daniela Cioboata ${ }^{2}$, Florina Doandes², Delia Nicoara², \\ Mihaela Dobre ${ }^{2}$, Marioara Boia ${ }^{1,2}$ \\ ${ }^{1}$ Neonatology Department, "Victor Babes" University of Medicine and Pharmacy, \\ Timisoara \\ 2 “Louis Turcanu” Children's Emergency Hospital, Timisoara
}

\begin{abstract}
Aim. The main purpose is to evaluate the predisposing factors, the evolution and the associated pathology of the pneumothorax in the neonatal period.

Materials and methods. Retrospective study over two years between 2014 and 2015 on 11 patients hospitalized in the Neonatology departement of "Louis Turcanu" Children's Emergency Hospital, Timisoara, diagnosed with pneumothorax.

Results. From 1689 newborn infants admitted, 11 patients developed pneumothorax, the incidence was $0.65 \%$. 8 patients $(73 \%)$ were males and $3(27 \%)$ were females. 4 out of those 11 patients were term newborn infants (gestational age $>37$ weeks) and 7 patients were premature newborns ( $<37$ weeks of gestational age). Based on the birth weight 4 patients had over 2,500 grams and 7 under 2,500 grams. The main cause of the pneumothorax in the study group was the respiratory distress syndrome $(45,4 \%)$.

Conclusions. Pneumothorax represents an important condition in the neonatal pathology, it is essential to recognize and initiate the treatment from early stages to reduce the complications.
\end{abstract}

Keywords: pneumothorax, newborn

\section{INTRODUCTION}

Pneumothorax is defined by the presence of air between the parietal and visceral pleura.

The incidence is $1-2 \%$ in the newborn population, with a frequency of $5-7 \%$ in premature newborn infants with a weight under 1,500 grams $(3,4)$. Pneumothorax can occure spontaneousely with the appeareance of air collection in the absence of intubation, possitive pressure ventilation or due to some associated respiratory diseases $(7,8)$. Respiratory distress syndrome, meconium aspiration, pulmonary hypoplasia, neonatal sepsis, congenital pneumony associated or not with reanimation maneuvres at birth and/or mechanical ventilation are risk factors for secondary pneumotorax $(4,6)$.

The main objective of the study is to evaluate the predisposing factors, the evolution and associated pathology of the pneumothorax in the neonatal period. The secondary objective aimed to analyze the clinical and evolutive particularities in the patients diagnosed with the pneumothorax.

\section{MATERIAL AND METHODS}

Retrospective study over 2 years from January 2014 to December 2015 which analyzed the data from 11 patients admitted to "Louis Turcanu" Children's Emergency Hospital, Neonatology department, Timisoara, diagnosed with pneumothorax. The diagnose was established based on the anamnestic data, clinical examination, blood gas analysis (hypoxia and respiratory acidosis) and chest X-ray.

The main method of data reception was analyzing the clinical observation sheet, including data about gestational age, birth weight, Apgar score, the cause of pneumothorax and associted comorbidities.

\section{RESULTS}

From the 1,689 newborn infants admitted between 2014-2015, 11 patients developed pneumothorax with an incidence of $0.65 \%$ and a prevalence of 5\% (admitted to Neonatal Intensive Care Unit).

Corresponding author:

Assoc. Prof. Marioara Boia, MD, PhD, "Victor Babes” University of Medicine and Pharmacy, 2 Murgu Eftimie Pta, Timisoara

E-mail: marianaboia@yahoo.com 
Distribution of cases by sex revealed a significant increase percentage in male patients -8 newborns $(73 \%)$ as opposed to female patients $-3(27 \%) .4$ out of those 11 patients were term newborn infants (gestational age $>37$ weeks) and 7 patients were premature newborns $(<37$ weeks of gestational age). Based on the birth weight, 3 premature infants $(42,8 \%)$ had a birth weight under 1,500 grams and 8 premature infants $(57.2 \%)$ had a birth weight between 1,500 2,500 grams. The average Apgar score in the study group was 5 (2-8) (Table 1).

TABLE 1. The analysis of pneumothorax risk factors

\begin{tabular}{|c|c|c|}
\hline & Total & Death rate \\
\hline \multicolumn{3}{|l|}{ APGAR Score } \\
\hline Under 5 & $8(72.3 \%)$ & $2(18.2 \%)$ \\
\hline Over 5 & $3(27.7 \%)$ & $0(0 \%)$ \\
\hline \multicolumn{3}{|l|}{ Gestational age } \\
\hline Over 37 weeks & $4(36.4 \%)$ & $1(9.1 \%)$ \\
\hline Under 37 weeks & $7(63.6 \%)$ & $2(27.7 \%)$ \\
\hline \multicolumn{3}{|l|}{ Birth weight } \\
\hline Over $2,500 \mathrm{~g}$ & $4(36.4 \%)$ & $1(9.1 \%)$ \\
\hline Under 2,500 g & 7 (63.6\%) & $2(27.7 \%)$ \\
\hline
\end{tabular}

From a total of 11 cases, 3 developed by bilateral pneumothorax (27.3\%), 6 cases developed right pneumothorax $(54.5 \%)$ and 2 cases with left pneumothorax (18.2\%).

The most frequent cause of pneumothorax was respiratory distress syndrome followed by pneumonia and mechanical ventilation (Table 2).

TABLE 2. Pneumothorax causes

\begin{tabular}{|l|c|}
\hline & $\mathrm{n}(\%)$ \\
\hline Respiratory distress syndrome & $5(45.4 \%)$ \\
\hline Pneumonia & $2(18.2 \%)$ \\
\hline Mechanical ventilation & $2(18.2 \%)$ \\
\hline Birth reanimation & $1(9.1 \%)$ \\
\hline Spontaneous pneumothorax & $1(9.1 \%)$ \\
\hline
\end{tabular}

The most frequent complications encountered in the studied cases were: pulmonary hypertension $40 \%$ of cases, intraventricular hemorrhage $-20 \%$ of cases, chronic lung disease $-20 \%$ of patients, pulmonary hemorrhage $-10 \%$, sepsis $-10 \%$. Death occured in 3 cases, correlated with small gestational age, birth weight under 2,500 grams and Apgar score under 5 according to Table 1.

In $10 \%$ of followed cases, a spontaneous remission occured, while $90 \%$ of patients needed surgical aspirative drainage (Fig. 1). Subsequentely, all patients needed mechanical ventilation.
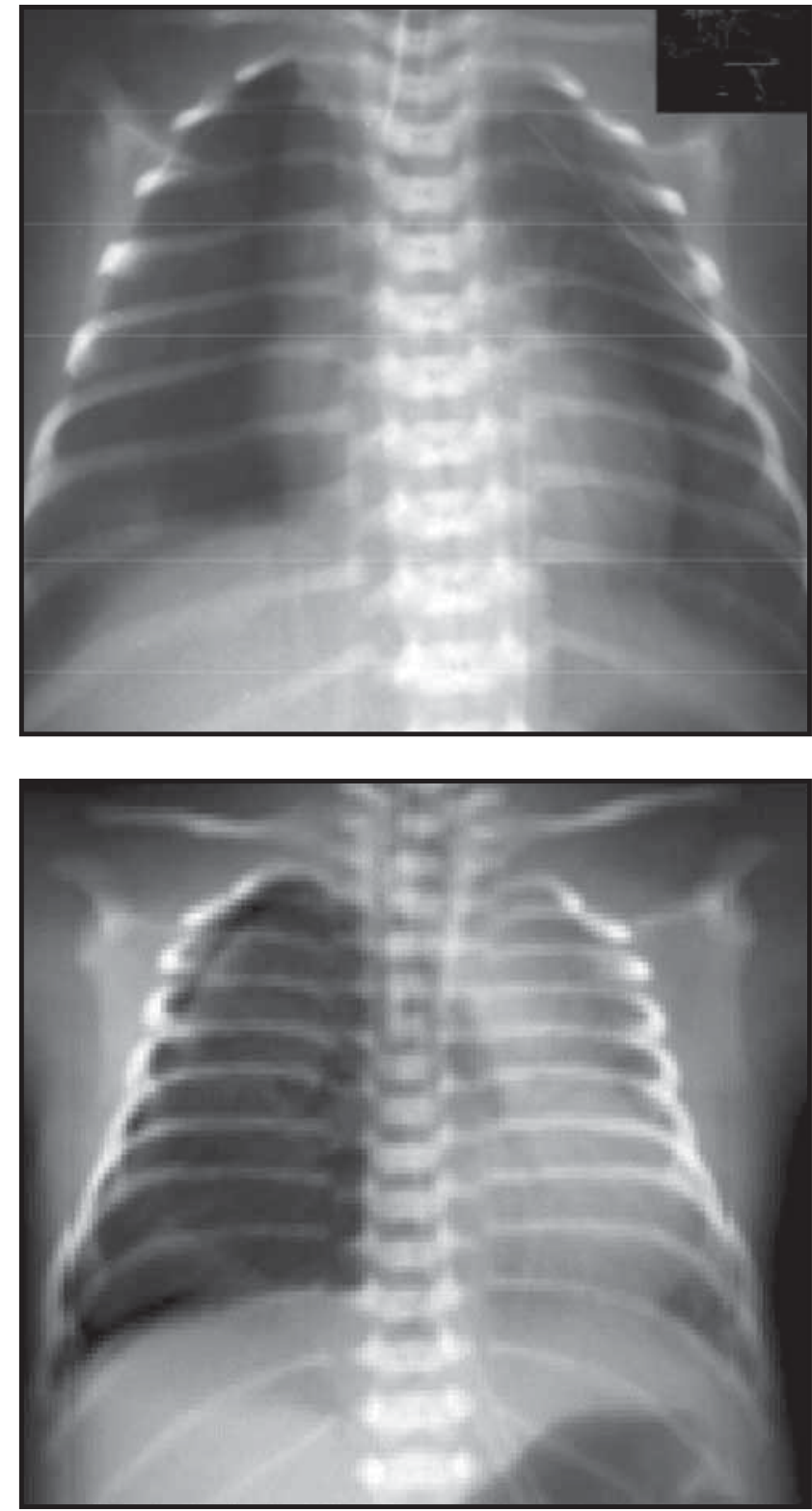

FIGURE 11. Left lung pleural drainage and right pneumothorax with mediastinum pushed to the left

\section{DISCUSSIONS}

Pneumothorax is more frequently in newborn infants, particulary premature infants than other age groups. Pneumothorax incidence in speciality literature is $0.5-1 \%$ in term infants, with an increased percentage at 13 in premature infants with a weight between 500-700 grams, while the incidence in newborns with birth weight between $1,250-1,500$ grams is approximately $2 \%$ (5). In the study group, the premature infants weighing between 1,000-1,500 grams presented an increased risk in developing pneumothorax, representing approximately $64 \%$ of cases. Low birth weight, small Apgar score and small gestational age are predictive factors for pneumothorax in studied patients. 
Newborn infants with meconium aspiration, respiratory distress syndrome whom required ressuscitation maneouvers at birth have a higher incidence in developing pneumothorax. In some speciality literature, studies was highlighted that newborn infants that required mechanical ventilation with high inspiratory pressure or continuous possitive and expiratory pressure, may have $26 \%$ incidence in developing a pneumothorax (2). In the study group, the main cause was represented by the respiratory distress syndrome $-45 \%$ followed pneumonia and mechanical ventilation in an equal percentage of approximately $18 \%$. Often in premature patients with respiratory distress syndrome, pneumothorax may occur once with the regresion of the disease and increased pulmonary compliance, as well as the lack of reducing the ventilation parameters.

The morbidity and mortality rate in the pneumothorax in the neonatal period is approximately 2325\% linked to patients with low Apgar score and premature infants who associate respiratory conditions $(1,4)$. In our study, death occured in 3 cases, 2 of them were premature infants and 1 was term infant. 2 of deceased cases had an Apgar score under 5 and a weight under 2,500 grams, obtained data were correlated with the data from speciality literature.

\section{CONCLUSION}

Pneumothorax represents an important condition in the neonatal pathology. An increased incidence is been in male patients, compared with female patients. Premature patients have a higher risk to develop pneumothorax because of the imaturity of the respiratory system.

In order to reduce the risk of developing pneumothorax, it is highly necessary to be made a rigurous control of the ventilation parameters including optimizing the possitive respiratory pressure and decreasing the peak inspiratory pressure.

It is essential to recognize and initiate the treatment from early stages in order to reduce the complication secondary to hypoxia, hypercapnia.

\section{REFERENCES}

1. Brunherotti M., Vainna J., Silveira C. Decrease of the occurence of pneumothorax in newborns with respiratory distress syndrome through reduction of ventilatory parameters. J Pediatr (Rio J) 2003; 79 (1): 75-80.

2. Jeng M.J., Lee Y.S., Tsao P.C. et al. Neonatal air leaksyndrome and role of high-frequency ventilation in its prevention. Journal of the Chinese Medical Association 2012; 75: 551-559.

3. Esme H., Dogru O., Eren S. et al. The factors affecting persistent pneumothorax and mortality in neoantal pneumothorax. The Turkish Journal of Pediatrics 2008; 50: 242-246.

4. Dordevic I., Slavkovic A., Slavkovic-Jovanovic M. et al. Influence of risk factors on frequency and prognosis of neonatal pneumothorax, five-year experience. Acta MedicaMedianae 2010; 49 (2): 5-8. 